\title{
Comparative transcriptomic analysis of Lactiplantibacillus plantarum RS66CD biofilm in high-salt conditions and planktonic cells
}

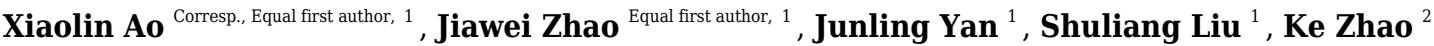 \\ ${ }^{1}$ college of food science, Sichuan Agricultural University, ya'an, Sichuan, China \\ 2 \\ 2 Colloge of resources, Sichuan agricultural university, cheng'du', China \\ Corresponding Author: Xiaolin Ao \\ Email address: huavslin@163.com
}

BACKGROUND: Lactiplantibacillus plantarum (L. plantarum), a dominant strain in traditional fermented foods, is widely used in fermentation industry because of its fast acid production. However, L. plantarum is easily inactivated due to acidity, high temperature and other factors. The formation of biofilm by bacteria can effectively increase environmental tolerance. Therefore, it is important to improve the environmental tolerance of $L$. plantarum by studying its biofilm formation conditions and regulatory mechanisms.

METHODS: After determining a suitable $\mathrm{NaCl}$ concentration for promoting biofilm formation, $L$. plantarum was grown with $48 \mathrm{~g} \mathrm{~L}^{-1} \mathrm{NaCl}$. Differential gene expressions in L. plantarum biofilm vs. planktonic cells were analyzed using RNA sequencing and validated using qPCR.

RESULT: L. plantarum RS66CD biofilm formation formed highest amount of when grown at $48 \mathrm{~g} \cdot \mathrm{L}^{-1} \mathrm{NaCl}$. Altogether 447 genes were up-regulated and 426 genes were down-regulated in the biofilm. KEGG pathway analysis showed that genes coding for D-Alanine metabolism, peptidoglycan biosynthesis, twocomponent system, carbon metabolism, bacterial secretion system, lysine biosynthesis and fatty acid metabolism were crucial for biofilm formation. In addition, eight other genes related to biofilm formation were differentially expressed. Our results provide insights into the differential gene expression involved in biofilm formation, which can help to reveal gene regulation during L. plantarum biofilm formation. 
1 Comparative transcriptomic analysis of Lactiplantibacillus plantarum RS66CD

2 biofilm in high-salt conditions and planktonic cells

3 Xiaolin Ao*\&1, Jiawei Zhao ${ }^{\& 1}$, Junling Yan ${ }^{1}$, Shuliang Liu¹, Ke Zhao

$4 \quad \&$ Xiaolin Ao and Jiawei Zhao contributed equally to this work. Author order was determined

5 both alphabetically and in order of increasing seniority.

61 Colloge of food science, Sichuan Agricultural University, Ya'an, Sichuan, China

72 Colloge of resources, Sichuan Agricultural University, Ya'an, Sichuan, China

$8 *$ Corresponding author:

9 Xiaolin $\mathrm{Ao}^{1}$

1046 Xinkang Road, Ya’an, Sichuan, 625000, China

11 E-mail: huavslin@163.com

12 


\section{Abstract}

14

BACKGROUND: Lactiplantibacillus plantarum (L. plantarum), a dominant strain in traditional fermented foods, is widely used in fermentation industry because of its fast acid production. However, L. plantarum is easily inactivated due to acidity, high temperature and other factors. The formation of biofilm by bacteria can effectively increase environmental tolerance.

Therefore, it is important to improve the environmental tolerance of $L$. plantarum by studying its biofilm formation conditions and regulatory mechanisms.

METHODS: After determining a suitable $\mathrm{NaCl}$ concentration for promoting biofilm formation, L. plantarum was grown with $48 \mathrm{~g} \mathrm{~L}^{-1} \mathrm{NaCl}$. Differential gene expressions in L. plantarum biofilm vs. planktonic cells were analyzed using RNA sequencing and validated using qPCR.

RESULT: L. plantarum RS66CD biofilm formation formed highest amount of when grown at $48 \mathrm{~g} . \mathrm{L}^{-1} \mathrm{NaCl}$. Altogether 447 genes were up-regulated and 426 genes were down-regulated in the biofilm. KEGG pathway analysis showed that genes coding for D-Alanine metabolism, peptidoglycan biosynthesis, two-component system, carbon metabolism, bacterial secretion system, lysine biosynthesis and fatty acid metabolism were crucial for biofilm formation. In addition, eight other genes related to biofilm formation were differentially expressed. Our results provide insights into the differential gene expression involved in biofilm formation, which can help to reveal gene regulation during L. plantarum biofilm formation.

Key words: Lactiplantibacillus plantarum; Biofilm; Transcriptomics; Gene expression

\section{Introduction}

Lactiplantibacillus plantarum, a facultative heterofermentative lactobacilli, is commonly used in fermenting vegetables (Alan Y et al., 2018), cereals (Oguntoyinbo F A., et al., 2015), fermented dairy products 
34 (Zhang SS et al., 2020). In addition, L. plantarum could be linked with some health effects. For example,

clinical studies have found that L. plantarum CECT7315 and CECT7316 can enhance the immunity of the

elderly (Mane et al., 2011). The survivability of L. plantarum has been found to be influenced by many factors, e.g. heat treatment, storage temperature and time, acid, microbiota competition and possible presence of bacteriocins or other antimicrobials (Mona et al., 2020, Tripathi et al., 2014). Many microorganisms resist the growth-unfriendly environment by forming biofilms (Wu et al., 2019). Therefore, we aimed at improving the environmental tolerance of $L$. plantarum during fermentation and storage by inducing biofilm formation.

Biofilms are composed of microorganisms and an extracellular polymeric matrix that contain polysaccharides, proteins, nucleic acids, and lipids (Flemming et al., 2010). Biofilm can effectively increase the stress tolerance of cells. L. plantarum JCM 1149 had greater resistance to organic acids, ethanol and sodium hypochlorite inside biofilm than inside planktonic cells (Kubota et al., 2009). The biofilm-forming Lacticaseibacillus rhamnosus not only showed greater heat resistance, but also maintained higher activity in simulated gastrointestinal tract environment (Kiew et al., 2014). Because the formation of biofilms effectively improves the resistance of microorganisms to extreme environments, studying the mechanism of biofilm formation has become a hot topic.

The formation of biofilms is affected by many factors, such as the roughness of the surface of the carrier, $\mathrm{pH}$, adhesion and gene regulation (Cappitelli et al., 2014). Also, inorganic ions affect the formation of biofilms. $\mathrm{Mn}^{2+}$ promoted biofilm formation in L. plantarum by eliminating oxidative stress and stimulating the secretion of more extracellular polymer substances (EPS) (Ibusquiza et al., 2015). The formation of the initial biofilm of Pseudomonas fluorescens can be promoted by $\mathrm{Mg}^{2+}$, yet higher concentrations lead to blockage of mass transfer channels, affecting the intake of nutrients and inhibiting the further development of biofilm (Song et 
55

56

57

58

59

60

61

62

63

64

65

66

67

68

69

70

71

72

73

74

75

al., 2006, Hoyle et al., 1992). $\mathrm{Fe}^{3+}$ promoted the secretion of EPS and increased the expression level of genes to improve the stability of biofilms in Staphylococcus epidermidis (Oliveira et al., 2017). Salt stress by NaCl or $\mathrm{KCl}$ promoted biofilm formation in L. plantarum and compensated for biofilm phenotypic instability, possibly by regulating the expression of biofilm formation related genes (Valle et al., 2007, Rachid et al., 2000). In fermenting vegetables, $6 \%-8 \%$ of sodium chloride is commonly applied to improve the flavor and inhibit the growth of spoilage microorganisms (Xiong T et al., 2012, Zhang Q S et al., 2016, Chun BH., 2020). Therefore, it is relevant to study the biofilm formation of L. plantarum in this concentration range.

In recent years, molecular and post-genomic analyses have reported in detail genes specific to the formation of biofilm. For instance, RNA-Seq analysis of Enterococcus faecalis isolates identified 163 differentially regulated genes in biofilm compared with planktonic cells, and that of Salmonella Typhimurium showed that 618 genes were over-expressed and 897 genes down-expressed in biofilm compared with planktonic cells (Seneviratne et al., 2017). In the biofilm formation of Bacillus amylobacillus, the antimicrobial peptide gene $l c i$ and the drug resistance gene $y v q H I$ were among the most up-regulated genes in induction of systemic resistance (Kröber et al., 2016). RNA-seq results showed that added tea tree oil caused differential expression of 304 genes in Staphylococcus aureus biofilms compared to planktonic cells (Zhao et al., 2018). Although biofilms made by pathogens have been studied extensively, analyses of L. plantarum biofilm formation are still scarce. Therefore, we studied the differential genes during the formation of $L$.

plantarum RS66CD biofilm at high salt concentration by transcriptomics.

\section{Materials and methods}

\subsection{The culture conditions and determination of biofilm formation}

L. plantarum RS66CD (GenBank MN160322) isolated from traditional Sichuan paocai has good 
fermentation ability. Previous experimental results showed that L. plantarum RS66CD was able to form biofilm (Zhao et al., 2019). Therefore, L. plantarum RS66CD was selected as the experimental strain. We hope to promote the biofilm formation of $L$. plantarum under the condition of $\mathrm{NaCl}$ concentration of $4 \%-6 \%$ (traditional Sichuan paocai fermentation), so as to improve the bacterial salt tolerance. In this experiment, the biofilm formation of $L$. plantarum $\mathrm{RS} 66 \mathrm{CD}$ was tested by growing it in modified, $\mathrm{MgSO}_{4}$ and $\mathrm{MnSO}_{4}$-free MRS medium with 40, 44, 46, 48, 52, 56 or 60 g.L.-1 $\mathrm{NaCl}$ in 96-well cell culture plates at $37^{\circ} \mathrm{C}$. After $24 \mathrm{~h}$ incubation, the culture solution was discarded, and biofilms were stained with $50 \mu \mathrm{L}$ of $0.5 \%$ crystal violet for 5 to $10 \mathrm{~min}$, washed with sterile distilled water for 2 to 3 times, after which the plates were dried at $37^{\circ} \mathrm{C}$, and absorbencies were measured at $490 \mathrm{~nm}$.

The concentration of sodium chloride, at which the maximum amount of biofilm was formed, was used in subsequent experiments. Planktonic cells, which were cultured in MRS liquid medium at $37^{\circ} \mathrm{C}$ for $24 \mathrm{~h}$ (as control treatment) and biofilm formation cells were fixed in $2.5 \%$ glutaraldehyde phosphate buffer solution at $4{ }^{\circ} \mathrm{C}$ for $8 \mathrm{~h}$, after which water was removed with ethanol. After gold plating, samples were examined using SEM.

\subsection{RNA extraction, library preparation, and sequencing}

For RNA extraction, cells were collected using centrifugation after culturing at $37^{\circ} \mathrm{C}$ for $24 \mathrm{~h}$. Total RNA was extracted using TRIZOL Reagent according the manufacturer's instruction (Invitrogen, California, USA) and genomic DNA was removed using DNase I (TaKara, Shiga, Japan). The quality of RNA determined using 2100 Bioanalyser (Agilent, California, USA). RNA was quantified using the ND-2000 (NanoDrop Technologies). RNA-seq strand-specific libraries were prepared from $5 \mu \mathrm{g}$ of total RNA using TruSeq RNA sample 
97

preparation Kit from Illumina (San Diego, CA). Shortly, rRNA was removed using RiboZero rRNA removal kit (Epicenter, Stockholm, Swedish) and fragmented using fragmentation buffer. cDNA synthesis, end repair, A-base addition and ligation of the Illumina-indexed adaptors were performed according to the Illumina protocol. Libraries were size selected for cDNA target fragments of 200-300 bp on 2\% Low Range Ultra Agarose followed by PCR amplification using DNA polymerase (NEB) for 15 PCR cycles. After libraries were quantified by TBS380, 150 bp*2 paired-end sequencing was done on Illumina Novaseq 6000 . The transcriptome sequencing data was submitted to NCBI with the accession number PRJNA626519.

\subsection{Quality control and mapping}

The raw paired end reads were trimmed and quality controlled using Trimmomatic v.0.36 (http://www.usadellab.org) with sliding window set to length 4 and Phred quality score 15, and minimum length to 75 .The clean reads were separately aligned to the reference genome (Lactobacillus plantarum WCFS1, GenBank: AL935263) with orientation mode using Rockhopper software (http://cs.wellesley.eduedge/ btjaden/Rockhopper). The expression levels of genes were calculated using Rockhopper with default parameters.

\subsection{Differential gene expression analysis}

To identify differentially expressed genes (DEGs) in the biofilm vs. planktonic cells, the expression levels of the transcripts were calculated using the fragments per kilobase of read per million mapped reads (RPKM) method. EdgeR (https://bioconductor.org/packages/release/bioc/html/edgeR.html) was used for differential expression analysis. The DEGs were selected using the following criteria: the logarithmic of fold change was greater than 2 and the false discovery rate (FDR) less than 0.05 . To understand the functions of the DEGs, GO functional enrichment and KEGG pathway analysis were carried out using Goatools 
118

119

120

121

122

123

124

125

126

127

128

129

130

131

132

133

134

135

136

137

138

(https://github.com/tanghaibao/GOatools) and KOBAS (http://kobas.cbi.pku.edu.cn/), respectively. DEGs were significantly enriched in GO terms and metabolic pathways when their Bonferroni-corrected P-value was less than 0.05. DGEs in biofilm formation of L. plantarum RS66CD were further analyzed using hierarchical clustering and a heat map.

\section{5 qRT-PCR validation of the transcriptome data}

To validate the transcriptome data, six genes were selected for RT-PCR experiments with 16S rRNA gene as the internal reference (Table 1).Total RNA was extracted from L. plantarum RS66CD samples using RNA isolation reagent (TIANGEN, Beijing, China) and the first strand cDNA synthesis was carried out using FastKing RT kit (TIANGEN, Beijing, China) according to manufacturer's instructions. RT-PCR amplification using three biological replicates per gene was done in a reaction volume of $20 \mu \mathrm{L}$ containing $10 \mu \mathrm{l}$ of YBR Green Supermix, $0.4 \mu \mathrm{L}$ of forward primer, $0.4 \mu \mathrm{L}$ of reverse primer, $0.8 \mu \mathrm{L}$ of cDNA and $8.4 \mu \mathrm{L}$ of ddH $\mathrm{d}_{2} \mathrm{O}$. The qPCR conditions were as follows: predenaturation at $95{ }^{\circ} \mathrm{C}$ for $10 \mathrm{~min}$, denaturation at $95{ }^{\circ} \mathrm{C}$ for $15 \mathrm{~s}$, and annealing and extension at $60{ }^{\circ} \mathrm{C}$ for $60 \mathrm{~s}$; fluorescence signals were collected during annealing and extension and the whole process was repeated for 40 cycles. Melting curve analysis is $95^{\circ} \mathrm{C}$ for $10 \mathrm{~s}, 65^{\circ} \mathrm{C}$ for $5 \mathrm{~s}$, and $95^{\circ} \mathrm{C}$ for $50 \mathrm{~s}$.

\subsection{The statistical analyses}

All the experiments were conducted in triplicate. Data related to the amount of biofilm formation was tested using Duncan's multiple range test in SPSS 22.0 for Windows. All data were reported as mean \pm standard deviation. Differences were taken as statistically significant at $P<0.05$.

\section{Result}

\subsection{Formation of $L$. plantarum RS66CD biofilm}

PeerJ reviewing PDF | (2020:03:46860:2:1:NEW 3 Jul 2020) 
L. plantarum $\mathrm{RS} 66 \mathrm{CD}$ formed more biofilm with $48 \mathrm{~g} \cdot \mathrm{L}^{-1} \mathrm{NaCl}$ than with other $\mathrm{NaCl}$ concentrations

(Figure 1). Scanning electron microscopy (SEM) revealed that the cells grown in the modified MRS with $\mathrm{NaCl}$

were clearly embedded in an extracellular matrix and EPS caused the bacteria to aggregate to form

microcolonies (Figure 2), demonstrating that L. plantarum could form a distinct biofilm when grown with

$\mathrm{NaCl}$.

\subsection{Transcriptomic data for biofilm formation of $L$. plantarum RS66CD}

On the average, sequencing of the libraries from L. plantarum RS66CD biofilm and planktonic cells resulted in $17 \pm 13 * 10^{6}$ and $9.3 \pm 0.5 * 10^{6}$ clean reads, respectively, out of which $0.86 \pm 0.073 \%$ and $0.69 \pm 0.081 \%$ were assigned to rRNA (Table 2). The mapping ratio between sequencing samples and reference genome was above $80 \%$.

\subsection{Differential gene expression and KEGG pathway analysis}

The transcribed genes were classified into gene ontology (GO) categories: 1047 genes were classified into biological processes, 660 to cellular components and 208 to molecular functions. Compared to the planktonic cells, 447 genes were up-regulated and 426 genes were down-regulated in the biofilm (Figure 3).

The DEGs were compared with Kyoto Encyclopedia of Genes and Genomes (KEGG, http://www.genome.jp/kegg) and GO databases (http://www.geneontology.org). In the figure 4 the abscissa represents the enrichment factor; the vertical coordinate represents the enriched function of the GO term. The larger the circle is, the more differential genes are enriched to this function. Thirty metabolic pathways were enriched in the L. plantarum RS66CD biofilm (Figure 4). In D-alanine metabolism glutathione metabolism, bacterial secretion system, peptidoglycan biosynthesis, two-component system, and carbon metabolism pathways 27 genes were up-regulated and 12 down-regulated in the biofilm compared to the planktonic cells 
160

161

162

163

164

165

166

167

168

169

170

171

172

173

174

175

176

177

178

179

180

(Table 3, Figure 5). In addition, five biofilm related genes were up-regulated and four down-regulated in the biofilm (Table 4, Figure 5).

\section{3 qRT-PCR validation of differential expression}

Compared to planktonic cells the expression of groEL was 2.4 fold and 2.03 times higher in the biofilm as determined by qPCR and RNA-Seq, respectively, the expression of $f$ ts $H$ was 1.07 fold and 1.43 fold higher in biofilm, the expression of $g y r B$ was 0.96 and 0.83 fold higher, and the expression of alr 1.39 and 1.69 fold higher. For LlFabZ1 and LlFabZ2 (encode $f a b Z$ and regulation of ACP dehydratase and ACP dehydrated isomerase) the expression was 1.4 and 4.3 fold higher in biofilm, as determined by qPCR and RNA-Seq, respectively. As the qRT-PCR validation of differential expression was consistent with the RNA-Seq, the transcriptomics results were considered representative.

\section{Discussions}

To increase tolerance against adverse environmental conditions, bacteria form biofilms to protect the cells from external forces (Yan et al., 2019).The formation of biofilm has several probable mechanisms including hormone induced, environment stimulation, signaling and gene transfer (Ghafoor et al., 2011, Ramesh et al., 2019). The main mechanism of biofilm formation can be ascribed to the regulation of transcriptional bacterial physiological metabolism. Biofilms in industrial and clinical context have received considerable attention (Song et al., 2012). In this study we characterized the biofilm formation related gene expressionof $L$.

plantarum RS66CD.

\subsection{D-alanine and peptidoglycan biosynthesis}

In our study, D-alanine metabolism and peptidoglycan biosynthesis related genes were up-regulated in the RS66CD biofilm ( $d l t A, d l t C, d d l, a l r)$. D-alanine, a D-amino acid is abundantly present in the bacterial cell 
181

182

wall (Laura et al., 2014). D-alanine is a structural component in bacterial cell wall peptidoglycan. Bacteria modulate surface charge by attaching D-alanine esters to teichoic acids (Heptinstall et al., 1970), thus Dalanine affects the formation of biofilm.

Alanylation that increases the positive charge of teichoic acid is mediated by products of the $d l t$ genes (Nilsson et al., 2016). In the $d l t$ operon, $d l t A$ encodes d-alanine carrier protein ligase, and $d l t C$ a d-alanyl carrier proteinthat ensure the ligation of d-alanine to the d-alanyl carrier protein (Neuhaus and Baddiley., 2004). Strains lacking the $d l t$ operon have increased antibiotics and acid sensitivity (Boyd et al., 2000). Studies have shown that resistance to gentamicin was significantly reduced in the biofilm formed by Streptococcus mutans after the $d l t A$ was knockout (Nilsson et al., 2016). Interestingly, the reduced antibiotic tolerance of $d l t A$ mutants was not due to defects in biofilm formation, but rather to more negative charges on the mutant surface.

A future knockout experiment will reveal whether, the role of dltA of L. plantarum RS66CD is similar.

Alanine racemase (Alr) converts L-alanine to D-alanine by changing the stereochemical structure of the chiral $\alpha$-carbon atom of L-alanine, and participates in the synthesis of bacterial cell wall peptidoglycan (Tanner, 2002). $D d l$ is present in both Gram-negative and -positive bacteria and catalyzes the formation of D-Ala-D-Ala dipeptide in an early step in peptidoglycan synthesis, which can promote the synthesis of bacterial cell walls

(Ameryckx et al., 2018).

The $d a c A$ encoded D-alanine carboxypeptidase is a low molecular mass penicillin-binding protein (LMM PBP) (Despreaux er al., 1993). LMM PBPs may regulate cell morphology and mediate the formation of biofilm (Young., 2006). murA and murB in the mur enzyme family are involved in the synthesis of propeptides of peptidoglycans(UDP-MurNAc) and the regulation of bacterial osmotic pressure (Hrast et al., 2014, Hattersley et al., 2011). Our results imply that during the formation of L. plantarum RS66CD biofilm, the up- 
202

203

204

205

206

207

208

209

210

211

212

213

214

215

216

217

218

219

220

221

222

regulation of the peptidoglycan synthesis related genes promote the synthesis of peptidoglycan and improve the stability of the bacteria; plausibly, changes in the cell surface due to the up-regulation of the D-alanine biosynthesis related genes increase cell aggregation and promote the biofilm formation.

\subsection{Two-component system}

Two-component system is a signaling pathway that regulates the gene expression of bacteria and coordinates responses to environmental stimulus, mainly through adaptation to changes in the environment (osmolarity, light, temperature and oxygen) (Sieuwerts et al., 2010), and regulation of developmental pathways and behaviors (sporulation, biofilm formation, competence and chemotaxis) (Biondi., 2013).

In our study, the differentially expressed genes related to the two-component system included both upregulated (hytR, cit $G$, lrg operon, lam operon, $f t s H, l p-3125, m l e S)$ and down-regulated genes (pstF, cyd operon, $g \ln A, r p o N)$. The gene $c i t G$ is essential for holo-acyl carrier protein (ACP) synthesis in Escherichia coli and catalyzes the formation of a prosthetic group precursor from ATP and dephospho-CoA (Schneider et al., 2000). Bacterial ACPs are the donors of the acyl moiety involved in the biosynthesis of fatty acids, playing an important role in increasing the ability of form biofilm (Sílvia et al., 2008). $\operatorname{lrg} A$ and $\operatorname{lrg} B$ are presumably involved in the formation of the murein hydrolase transport channel to regulate signal transmission and then to control programmed cell death (Zhang et al., 2018). lamA, lamB and fts $H$ participates in the accessory gene regulator (AGR) quorum sensing system. The AGR system influences the formation of staphylococcal biofilms during the structuring and dispersion phases (Cheraghi et al., 2017). Fts $H$ gene may regulate adhesion proteins. Some studies have shown that the $f t s H$ gene increases the amount of biofilm formation and is related to multiple environmental stress responses (Bove et al., 2012). Further experiments with $f t s H$ mutant strain should reveal it affects the formation of biofilm by L. plantarum RS66CD and reduces the salt tolerance of the 
223 strain. The $\operatorname{agr}$ operon includes $\operatorname{agr} B, \operatorname{agr} D, \operatorname{agr} C$, and $\operatorname{agr} A$. $\operatorname{agr} B$ is regarded as a factor to regulate biofilm

224 dispersal, because biofilm biomass is inversely correlated with $a g r B$ expression (Li et al., 2018). $C y d A$ and

$225 c y d B$ regulate cytochrome bd ubiquinol oxidase formation. Cytochrome bd panthenol oxidase is a terminal

226 oxidase involved in aerobic respiration of prokaryotes (Hazan et al., 2016). It funnels electrons coming from

$227 \mathrm{NADH}$ and ubiquinol to cytochrome $c$, and it is also capable of producing significant amounts of the free

radical superoxide. L. plantarum lacks superoxide dismutase, thus it must reduce the formation of free radical superoxides to promote biofilm formation (Bazil, 2017). Due to changes in osmotic pressure, it can also lead to programmed cell death and decreased respiratory intensity. This may explain the down-regulation of $c y d A$ and

$c y d B$ and the up regulation of $l p \_3125$ and $m l e S$ that participate in aerobic respiratory metabolism and simultaneously reduced $\mathrm{NAD}(\mathrm{P})+$ to $\mathrm{NAD}(\mathrm{P}) \mathrm{H}$. These genes were enriched in this pathway mainly through sense external signal stimulation and influence the expression of quorum sensing system that leads to increases in the adhesion proteins thereby promoting the biofilm formation. This can resist the osmotic pressure changes caused by the addition of $\mathrm{NaCl}$. L. plantarum also reduced the production of free radical superoxide by regulating oxidase activity to promote the formation of biofilm and increase the salt tolerance.

\subsection{Carbon metabolism}

One the roles of carbon metabolism is to help bacteria to adapt to the environment when it changes. In our study, the differentially expressed genes related to the carbon metabolism included both up-regulated and down-regulated genes. The $l y t R$ family genes that encode putative proteins $F \operatorname{lm} A, F \operatorname{lm} B$, and $F \operatorname{lm} C$ were upregulated in the biofilm. The proteins might be involved in cell wall integrity, cell growth, and cell adhesion, and in the developmental process leading to biofilm formation (Muscariello et al., 2013). Given the important 
244 separately in future experiments. The genes $\operatorname{ser} A$ and $\operatorname{ser} C$ participate in the formation of phosphoserine

245 aminotransferase (P-Ser-HPr) that belongs to the carbohydrate phosphotransferase system. The inactivation of

246 Neisseria meningitidis hprK strongly diminished cell adhesion (Deutscher et al., 2005). serA and $\operatorname{serC}$ increase

247 the adhesion of strains by increasing the content of P-Ser-HPr, which promotes the formation of biofilm.

248 Pyruvate dehydrogenase( $\mathrm{Pdh})$ participates in many physiological processes, such as carbon metabolism,

249 aerobic respiration and fatty acid metabolism. $P d h A, P d h B$ and $P d h C$ are involved in the formation of a

250 pyruvate dehydrogenase complex which belongs to a family of alpha-keto-acid dehydrogenase complexes

251 (Neveling et al., 1998). In our study, the $p d h$ genes were down-regulated, which may be related to the change

252

253

254

255

256

257

258

259

260

261

262

263

264

in osmotic pressure inside biofilm, leading to a decrease in respiratory intensity and redox reactions, and to less

intense energy metabolism. Out of the other down-regulated genes pps gene catalyzes the phosphorylation of

pyruvate and participates in the TCA cycle (Smyer and Jeter, 1989). pgm5 gene regulates histidine phosphatase

that is mainly involved in the synthesis of ATP, and $t k t 4$ encodes a key enzyme of the non-oxidative branch of

the pentose phosphate pathway. NADPH participates in reductive biosyntheses and defense against oxidative

stress (Wood, 1986). These down-regulated genes reduce bacterial damage by improving the oxidative stress

response. The biofilm formation can be promoted to resist the change of osmotic pressure by enhancing the

bacterial adhesion, participating in the anabolism of serine which constitutes biofilm. Our study found that the

genes related to energy metabolism and respiratory intensity were inhibited due to the changes of osmotic

pressure.

\subsection{Bacterial secretion system}

Bacteria secrete many proteins to adapt to their living environment. Bacterial secretion system affects the formation of biofilm by regulating the entry and exit of substances which further affects energy metabolism

PeerJ reviewing PDF | (2020:03:46860:2:1:NEW 3 Jul 2020) 
265

266

267

268

269

270

271

272

273

274

275

276

277

278

279

280

281

282

283

284

285

and signal transmission. Protein export across the cell envelope of Gram-positive $(\mathrm{G}+)$ bacteria is relatively simple as only one membrane needs to be passed (Glöckner et al., 2008). In our study, three bacterial secretion system genes (traK, $\operatorname{rad} A$ and $\sec Y$ ) were up-regulated in the biofilm. Out of the up-regulated genes, traK encodes a transfer protein that regulates substance entry and exit and signaling. The $\mathrm{C}$ terminus of $\mathrm{rad} A$ is related to LonB protease, an serine protease that regulates capsular polysaccharide synthesis (Ishibashi et al., 2006). In many eubacterial $\mathrm{radA}$ orthologs, active-site serine residue is replaced by alanine, which may be related to the involvement of L-alanine in biofilm formation. SecY has been reported to be a transmembrane protein and the most important component of the Sec protein secretion system (Flower, 2007). The secY encodes a transmembrane protein in the general secretory $(\mathrm{Sec})$ pathway that is responsible for translocation of extracytoplasmic proteins across the plasma membrane in $\mathrm{G}+$ bacteria. The above genes regulate protein secretion to synthesize biofilm, and reduce the damage of osmotic pressure to strain through regulates substance entry and exit. Studies have shown that, the biofilms of L. plantarum were reduce the density by destroy extracellular matrix (Fernández Ramírez, et al., 2015), which further indicates that the formation of related proteins can promote biofilm formation.

\subsection{Other key genes}

Lysine can inhibit biofilm formation via inducing the production of reactive oxygen species and malondialdehyde (Ge et al., 2018). The genes in the Lysine biosynthesis pathway mainly inhibit the synthesis of redox-related enzymes and hydrolases (Table 4). Alcohol dehydrogenase are a group of oxidoreductases which encoded by lp_0862 gene during the stage of formation (Hu et al., 2019).

Fatty acids are the main components of cell membrane and participate in the physiological metabolism of bacteria. By regulating the type and composition of fatty acids, bacteria can transmit signals, regulate the 
286

287

288

289

290

291

292

293

294

295

296

297

298

299

300

301

302

303

304

305

306

fluidity of cell membranes, maintain membrane stability, and increase environmental tolerance. In our study,

four of the up-regulated genes ( $f a b G, f a b D, f a b H, f a b Z)$ were related to fatty acid metabolism, mainly involved

in the synthesis of type II fatty acids. The genes $f a b D$ and $f a b H$ encode ACP transacylase that catalyzes the formation of malonyl ACP, and 3-ketoacyl-ACP synthase III that catalyzes the condensation of malonyl ACP

and acetyl-CoA to form 3-keto butyryl ACP, respectively. The enzyme encoded by fabZ gene is mainly

involved in the cyclic reaction of fatty acid synthesis and promotes a new round of synthetic reactions. The

products of fatty acid synthesis include long-chain fatty acids, branched-chain fatty acids and unsaturated fatty

acids (UFA). Among them, long-chain fatty acids can be used as signal transduction substances (Zhou et al.,

2015). UFA has a low melting point which affects the physical properties of cell membranes, making them

important molecules in regulating bacterial cell membrane fluidity. Synthesizing branched-chain fatty acids is

a way to cope with unfavorable environments (Santiago et al., 2013). Comparison of biofilm components of

Staphylococcus aureus and Pseudomonas aeruginosaare with planktonic cells revealed that biofilm growth

mode leads to a decrease in the uneven-numbered chain phospholipids, and accumulation of long chain lipids

(Benamara et al., 2011, Rudy et al., 2019). This indicates that the accumulation of fatty acids could improve

the stability of biofilm. Combined with the results of RNA-Seq, it is shown that $L$. plantarum can affect the

fluidity of cell membrane and maintain the stability of biofilm by regulating the type and composition of fatty

acids, which can increase the environmental tolerance. Some studies have shown that $f a d D$ and $f a b H$ were

involved in fatty acid and lipid biosynthesis pathway during the formation of biofilm by L. plantarum DB200

(Angelis M D, et al., 2015). In addition, the heat stress protein (GroEL) also exhibited chloroprene and

immune modulatory-properties to improve the aggregation of bacteria and environmental resistance (Pessione.,

2012). In future experiments, the biofilm formation mechanism can be further analyzed by comparing the fatty

PeerJ reviewing PDF | (2020:03:46860:2:1:NEW 3 Jul 2020) 
307

308

309

310

311

312

313

314

315

316

317

318

319

320

321

322

323

324

325

326

327

acid contents on the surface of planktonic cells and biofilm forming strains.

In summary, this study provided important insights into gene expression during the formation of biofilm

by L. plantarum. With the formation of biofilm, transcriptome profile changed significantly. Specifically,

genes coding for D-alanine metabolism, peptidoglycan biosynthesis, two-component system, carbon

metabolism, bacterial secretion system and fatty acid metabolism were found to be crucial for biofilm

formation. The results will pave way for a more comprehensive understanding of the mechanisms involved in biofilm formation.

\section{Acknowledgements}

The authors acknowledge Associate Professor Petri Penttinen, Sichuan Agricultural University, for his

help in revising the manuscript.

\section{References}

Alan Y, Topalcengiz Z, Dığrak M. 2018. Biogenic amine and fermentation metabolite production assessments of Lactobacillus plantarum isolates for naturally fermented pickles. LWT 98: 322-328.

Ameryckx A, Thabault L, Pochet L, Leimanis S. 2018. 1-(2-Hydroxybenzoyl)-thiosemicarbazides are promising antimicrobial agents targeting D-alanine-D-alanine ligase in bacterio. European Journal of Medicinal Chemistry 159: 324-338.

Angelis M D, Siragusa S, Campanella D, Cagno RD. 2015. Comparative proteomic analysis of biofilm and planktonic cells of Lactobacillus plantarum DB200. Proteomics 15(13): 2244-2257.

Bazil JN. 2017. Analysis of a Functional Dimer Model of Ubiquinol Cytochrome c Oxidoreductase. Biophysical Journal 113: 1599-1612.

Benamara H, Rihouey C, Jouenne T, Alexandre S. 2011. Impact of the biofilm mode of growth on the inner 
328

membrane phospholipid composition and lipid domains in Pseudomonas aeruginosa. BBABiomembranes 1808(1): 98-105.

Biondi EG. Two-Component Systems[M]. 2013: 2116-2305.

Bove P, Capozzi P, Garofalo C, Rieu A, Spano G, Fiocco D. 2012. Inactivation of the ftsH gene of Lactobacillus plantarum WCFS1: effects on growth, stress tolerance, cell surface properties and biofilm formation. Microbiological Research 167(4): 187-193.

Boyd DA, Cvitkovitch DG, Bleiweis AS, Kiriukhin MY, Debabov DV, Neuhaus FC, Hamilton IR. 2000. Defects in D-alanyl-lipoteichoic acid synthesis in Streptococcus mutans results in acid sensitivity. Journal of bacteriology 182(21): 6055-6065.

Cao Q, Zhao Z, Zhang YZ, Wang Y, Ding JY. 2006. Cloning, sequence analysis and expression of alanine racemase gene in Pseudomonas putida. Acta Microbiologica Sinica 46(1): 80-84. (in China)

Cappitelli F, Polo A, Villa F. 2014. Biofilm Formation in Food Processing Environments is Still Poorly Understood and Controlled. Food Engineering Reviews 6(2014): 29-42.

Cheraghi S, Pourgholi L, Shafaati M, Fesharaki SH, Jalali A. 2017. Molecular Analysis of Virulence Genes and the accessory gene regulator (agr) types Among Methicillin Resistant Staphylococcus aureus (MRSA) Strains. Journal of Global Antimicrobial Resistance 10: 315-320.

Chun BH, Kim KH, Jeong SE, Jeon CO. 2020. The effect of salt concentrations on the fermentation of doenjang, a traditional Korean fermented soybean paste. Food Microbiology 86: 103329.

Despreaux CW, Manning RF. 1993. The dacA gene of Bacillus stearothermophilus coding for d-alanine carboxypeptidase: cloning, structure and expression in Escherichia coli and Pichia pastoris. Gene 131: $35-41$. 
349

350

351

352

353

354

355

356

357

358

359

360

361

362

363

364

365

366

367

368

369

Deutscher J, Herro R, Bourand A, Mijakovic I, Poncet S. 2005. P-Ser-HPr-a link between carbon metabolism and the virulence of some pathogenic bacteria. BBA-Proteins and Proteomics 1754(1-2): 118-125.

Fernández Ramírez M D, Smid E J, Abee T, Groot MNN. 2015. Characterisation of biofilms formed by Lactobacillus plantarum WCFS1 and food spoilage isolates. International journal of food microbiology 207(17): 23-29.

Flemming HC, Wingender J. 2010. The Biofilm Matrix. Nature Reviews Microbiology 8(9): 623-633.

Flower AM. 2007. The SecY translocation complex: convergence of genetics and structure. Trends Microbiol 15(5): 203-210.

Ge Y, Wei Y, Li C, Chen YR. 2018. Reactive oxygen species metabolism and phenylpropanoid pathway involved in disease resistance against Penicillium expansum in apple fruit induced by $\varepsilon$-poly-L-lysine. Journal of the Science of Food and Agriculture 98(13): 5082-5088.

Ghafoor A, Hay LD, Rehm BHA. 2011. Role of exopolysaccharides in Pseudomonas aeruginosa biofilm formation and architecture. Applied and Environmental Microbiology 77(15): 5238-5246.

Glöckner G, Weissenberger CA, Weinmann E, Jacobi S, Schunder E, Steinert M, Hacker J, Heuner K. 2008. Identification and characterization of a new conjugation/type IVA secretion system ( $\mathrm{tr}$ /tra) of Legionella pneumophila Corby localized on two mobile genomic islands. International Journal of Medical Microbiology 298(5-6): 411-428.

Hattersley GJ, Pérez-Velázquez J, Chappell MJ, Bearup D, Roper D, Dowson C, Bugg T, Evans ND. 2011. Indistinguishability and identifiability of kinetic models for the MurC reaction in peptidoglycan biosynthesis. Computer Methods \& Programs in Biomedicine 104(2): 70-80.

Hazan R, Que YA, Maura D, Strobel B, Majcherczyk PA, Hopper LR. 2016. Auto Poisoning of the 
Respiratory Chain by a Quorum-Sensing-Regulated Molecule Favors Biofilm Formation and Antibiotic Tolerance. Current biology 26(2): 195-206.

Heptinstall S, Archibald AR, Baddiley J. 1970. Teichoic Acids and Membrane Function in Bacteria. Nature 225: 519-521.

Hoyle BD, Wong CKW, Costerton JW. 1992. Disparate efficacy of tobramycin on $\mathrm{Ca}^{2+}, \mathrm{Mg}^{2+}$, and HEPES treated Pseudomonas aeruginosa biofilms. Canadian Journal of Microbiology 38(11): 1214-1218.

Hrast M, Sosič I, Šink R, Gobec S. 2014. Inhibitors of the peptidoglycan biosynthesis enzymes MurA-F. Bioorganic Chemistry 55: 2-15.

Hu ZH, Pu J, Bai YJ. 2019. Characterisation of five alcohol dehydrogenases from Lactobacillus reuteri DSM20016. Process Biochemistry 86: 73-79.

Ibusquiza PS, Groot MN, DEBÁN-Valles A, Abee T, Besten HMW. 2015. Impact of growth conditions and role of sigB on Listeria monocytogenes fitness in single and mixed biofilms cultured with Lactobacillus plantarum. Food Research International 71: 140-145.

Ishibashi T, Isogai M, Kiyohara H, Hosaka M, Chiku H, Koga A, Yamamoto T, Uchiyama Y, Mori Y, Hashimoto J, Ausió J, Kimura S, Sakaguchi K. 2006. Higher plant RecA-like protein is homologous to RadA. DNA Repair 5(1): 80-88.

Kiew TY, Cheow W S, Hadinoto K. 2014. Importance of biofilm age and growth medium on the viability of probiotic capsules containing Lactobacillus rhamnosus GG biofilm. LWT-Food Science and Technology 59(2): 956-963.

Kröber M, Verwaaijen B, Wibberg D, Winkler a, Pühler A, Schlüter A. 2016. Comparative transcriptome analysis of the biocontrol strain Bacillus amyloliquefaciens FZB42 as response to biofilm formation 
391

392

393

394

395

396

397

398

399

400

401

402

403

404

405

406

407

408

409

410

411

analyzed by RNA sequencing. Journal of Biotechnology 231(10): 212-223.

Kubota H, Senda S, Tokuda H, Tokuda H, Uchiyama H, Nomura N. 2009. Stress resistance of biofilm and planktonic Lactobacillus plantarum subsp. plantarum JCM 1149. Food Microbiology 26(6): 592-597.

Laura A, Akbar E, Hermoso JA, Pedro MA, Cava F. 2014. Peptidoglycan remodeling by the coordinated action of multispecific enzymes. Microbial Drug Resist 20(3): 190-198.

Li T, Li SR, Jiang B, Hu XM. 2018. Therapeutic Targeting of the Staphylococcus aureus Accessory Gene Regulator (agr) System. Frontiers in Microbiology 9: 55.

Lopez PM, Reina MR, Trier A, Herrfurth C, Feussner I, Bernal A, Forero M, Leidy C. 2019. Variations in carotenoid content and acyl chain composition in exponential, stationary and biofilm states of Staphylococcus aureus, and their influence on membrane biophysical properties. BBA- Biomembranes 1861(5): 978-987.

Mahmoud M, Abdallah N A, El-Shafei K, Tawfik NF. 2020. Survivability of alginate-microencapsulated Lactobucillus plantarum during storage, simulated food processing and gastrointestinal conditions. Helyon 6(3): e03451.

Mane J, Pedrosa E, Loren V, Gassull MA, Espadaler J, Cuñé J, Audivert S, Bonachera MA, Cabré E. 2011. A mixture of Lactobucillus plantarum CECT7315 and CECT7316 enhances systemic immunity in elderly subjects. A dose-response, double-blind, placebo-controlled, randomized pilot trial. Nutricion Hospitalaria 26: 1611-1613.

Ma JC, Luo B, Hu Z, Wang HH. 2014. Identification and function research of fabZ1 and fabZ2 of Lactococcus lactis. Progress in Biochemistry and Biophysics 41(8): 777-786.

Muscariello L, Marino C, Capri U, Vastano V, Marasco R, Sacco M. 2013. CcpA and three newly identified

PeerJ reviewing PDF | (2020:03:46860:2:1:NEW 3 Jul 2020) 
412

413

414

415

416

417

418

419

420

421

422

423

424

425

426

427

428

429

430

431

432

proteins are involved in biofilm development in Lactobacillus plantarum. Journal of basic microbiology 51(1): 62-71.

Neuhaus FC, Baddiley J. 2004. A Continuum of Anionic Charge: Structures and Functions of D-alanylteichoic acids in Gram-Positive Bacteria. Microbiology and Molecular Biology Reviews 67: 686-723.

Neveling U, Klasen R, Bringer MS, Sahm H. 1998. Purification of the pyruvate dehydrogenase multienzyme complex of Zymomonas mobilis and identification and sequence analysis of the corresponding genes. Journal of Bacteriology 180(6):1540-1548.

Nilsson M, Rybtke M, Givskov M, Høiby N, Twetman S, Nielsen TT. 2016. The dlt genes play a role in antimicrobial tolerance of Streptococcus mutans biofilms. International journal of antimicrobial agents 48(3): 298-304.

Oguntoyinbo FA, Narbad A. 2015. Multifunctional properties of Lactobacillus plantarum strains isolated from fermented cereal foods. Journal of Functional Foods 17: 621-631.

Oliveira F, France Â, Ccerca N. 2017. Staphylococcus epidermidis is largely dependent on iron availability to form biofilms. International Journal of Medical Microbiology 307(8): 552-563.

Rachid SK, Ohlsen U, Wallner J, Hacker J, Hecker M, Ziebuhr W. 2000. Alternative transcription factor sigma $\mathrm{B}$ is involved in regulation of biofilmexpression in a Staphylococcus aureus mucosal isolate. Journal of bacteriology 182(32): 6824-6826.

Ramesh S, Sivasubramanian S, Veera RA, Karutha PS. 2019. Synergistic antibiofilm efficacy of undecanoic acid and auxins against quorum sensing mediated biofilm formation of luminescent Vibrio harveyi. Aquaculture 498: 162-170.

Pessione E. 2012. Lactic acid bacteria contribution to gut microbiota complexity: lights and shadows. Frontiers 
433

434

435

436

437

438

439

440

441

442

443

444

445

446

447

448

449

450

451

452

453

in cellular and infection microbiology 2(86): 1-12.

Santiago B, Marek M, Faustoferri R.C, Quivey RG. 2013. The Streptococcus mutans aminotransferase encoded by $i l v E$ is regulated by $\operatorname{Cod} Y$ and $C c p A$. Journal of bacteriology 195(16): 3552-3562.

Seneviratne CJ, Suriyanarayanan T, Swarup S, Chia KHB, Nagarajan N, Zhang C. 2017. Transcriptomics Analysis Reveals Putative Genes Involved in Biofilm Formation and Biofilm-associated Drug Resistance of Enterococcus faecalis. Journal of Endodontics 43(6): 949-955.

Schneider K, Dimroth P, Bott M. 2000. Identification of triphosphoribosyl-dephospho-CoA as precursor of the citrate lyase prosthetic group. FEBS Letters 483: 165-168.

Sieuwerts S, Molenaar D, Sacha AFT, Beerthuyzen M, Stevens MJ, Janssen PWA, Ingham CJ, de Bok FA, de Vos WM, van Hylckama Vlieg JE. 2010. Mixed-culture transcriptome analysis reveals the molecular basis of mixed-culture growth in Streptococcus thermophiles and Lactobacillus bulgaricus. Applied and Environmental Microbiology 76: 7775-7784.

Sílvia AS, Ramos CG, Almeida F, Luís MS, Wopperer J. 2008. Burkholderia cenocepacia J2315 acyl carrier protein: a potential target for antimicrobials' development. Microbial Pathogenesis 45(5-6): 331-336.

Smyer JR, Jeter RM. 1989. Characterization of phosphoenolpyruvate synthase mutants in Salmonella typhimurium. Archives of Microbiology 153: 26-32.

Song B, Leff LG. 2003. Influence of magnesium ions on biofilm formation by Pseudomonas fluorescens. Microbiological Research 161: 355-361.

Song LY, Wu JF, Xi CW. 2012. Biofilms on environmental surfaces: evaluation of the disinfection efficacy of a novel steam vapor system. American journal of infection control 40(10): 926-930.

Tanner ME. 2002. Understanding nature's strategies for enzyme-catalyzed racemization and epimerization.

Peer) reviewing PDF | (2020:03:46860:2:1:NEW 3 Jul 2020) 
Accounts of chemical research 35(3): 237-246.

455

456

457

458

459

460

461

462

463

464

465

466

467

468

469

470

471

472

473

474

Tripathi M.K, Giri S.K. 2014. Probiotic functional foods: Survival of probiotics during processing and storage. Journal of Functional Foods 9(1): 225-241.

Valle J, Vergarairigaray M, Merino N, Penadés JR, Lasa I. 2007. $\sigma B$ Regulates IS256-Mediated Staphylococcus aureus Biofilm Phenotypic Variation. Journal of Bacteriology 189(7): 2886-2896.

Wood T. 1968. Physiological functions of the pentose phosphate pathway. Cell Biochemistry \& Function 4(4): $241-247$

Wu YC, Cai P, Jing XX, Jing XX, Niu XK. 2019. Soil biofilm formation enhances microbial community diversity and metabolic activity. Environment International 132: 105-116.

Xiong T, Guan QQ, Song SH, Hao MY, Xie MY. 2012. Dynamic changes of lactic acid bacteria flora during Chinese sauerkraut fermentation. Food Control 26(1): 178-181.

Yan J, Bonine LB. 2019. Surviving as a Community: Antibiotic Tolerance and Persistence in Bacterial Biofilms. Cell Host \&Microbe 26: 15-21.

Young KD. 2006. The selective value of bacterial shape. Microbiology and Molecular Biology Review 70: 660-703.

Zhang F, Gao J, Wang B, Huo DX, Wang ZX, Zhang JC, Shao YY. 2018. Whole-genome sequencing reveals the mechanisms for evolution of streptomycin resistance in Lactobacillus plantarum. Journal of Dairy Science 101(4): 2867-2874.

Zhang QS, Chen G, Shen WX, Wang Y, Zhang WX, Chi YL. 2016. Microbial safety and sensory quality of instant low-salt Chinese paocai. Food Control 59: 575-580.

Zhang SS, Xu ZS, Qin LH, Kong J. 2020. Low-sugar yogurt making by the co-cultivation of Lactobacillus

PeerJ reviewing PDF | (2020:03:46860:2:1:NEW 3 Jul 2020) 
475 plantarum WCFS1 with yogurt starter cultures. Journal of dairy science 103: 3045-3054.

476 Zhou L, Yu YH, Chen XP, Diab A, Wang HH, He YW. 2015. The Multiple DSF-family QS Signals are

477 synthesized from Carbohydrate and Branched-chain Amino Acids via the FAS Elongation Cycle.

$478 \quad$ Scientific reports 5: 13294.

479

Zhao JW, Ao XL, Cai YM, Liu SL, Chen AJ, Wan H, Xu F, Wang F, He JY. 2019. Effect of metal ions on the biofilm formation and environmental tolerance of Lactobacillus Plantarum RS66CD. FOOD AND FERMENTATION INDUSTRIES 45(21): 46-52. (in China)

Zhao XC, Liu ZH, Liu ZJ, Meng RZ. 2018. Phenotype and RNA-seq based transcriptome profiling of Staphylococcus aureus biofilms in response to tea tree oil. Microbial Pathogenesis 123: 304-313. 


\section{Table 1 (on next page)}

Table 1 Genes and primers in the RT-PCR 
1

\begin{tabular}{lll}
\hline Name & Primer sequence (5'-3') & Function \\
\hline 16S rRNA & F-TGAGTGAGTGGCGAACTGGTG & KT025937 \\
& R-GCCGTGTCTCAGTCCCAATG & \\
groEL & F-GCAAATCGCTTCTGTATCTTC & Chaperonin GroEL(KJ807061) \\
& R-CGCTTCCATCCTTATCATTGTC & \\
fts $H$ & F- ACGACTAATGTGAGTGTTGCTGAA & ATP-dependent metallopeptidase HflB \\
& R-ATCCTGCGATGATGGTGTGG & (MTU86407) \\
gyrB & F-AGAAGAGGAAGTTAGAGAAGA & DNA topoisomerase (ATP-hydrolyzing) \\
& R-GCATATCCACTGTTATATTGAAG & subunit B (MF988195) \\
\multirow{2}{*}{ alr } & F- & Alanine racemase (Cao Q et al, 2006) \\
& TAGGATCCATCGAACTCAAACACACCTGCGTC & \\
& R- & \\
& CGAAGCTTTGGCAATTTCAGTCGACGAGTATC & \\
LlFabZ2 & F- TAACATATGACTAAAAAATACGC & Regulation of ACP dehydratase \\
& R-CAGAAGCTTCAATGCCACATTGC & (Ma JC et al, 2014) \\
& R-GAACATATGACTGAAGTAAACATTAATG & ACP dehydrated isomerase \\
\hline
\end{tabular}

2 
Table 2 (on next page)

Table 2 RNA sequencing results for $L$. plantarum RS66CD biofilm and planktonic cells 
Table 2 RNA sequencing results for L. plantarum RS66CD biofilm and planktonic cells

\begin{tabular}{lccccccc}
\hline & \multicolumn{3}{c}{ L. plantarum RS66CD biofilm } & & \multicolumn{2}{c}{ Planktonic cells } & \\
\cline { 2 - 3 } \cline { 7 - 8 } & Replicate 1 & Replicate 2 & Replicate 3 & & Replicate 1 & Replicate 2 & Replicate 3 \\
\hline Clean reads & 8581850 & 10660184 & 32053428 & & 9674730 & 9630650 & 8713450 \\
Clean base & 1281768285 & 1591930909 & 4765116422 & & 1444240232 & 1436102731 & 1301244000 \\
rRNA (\%) & 0.92 & 0.89 & 0.78 & & 0.65 & 0.63 & 0.78 \\
Mapped reads & 7038442 & 8769430 & 26643418 & & 7805908 & 7837808 & 6956864 \\
Mapped rate(\%) & 82.02 & 82.26 & 83.12 & & 80.68 & 81.38 & 79.84 \\
\hline
\end{tabular}

2 


\section{Table 3(on next page)}

Table 3 Differentially expressed genes in biofilm formation related KEGG pathways in $L$. plantarum RS66CD biofilm compared to planktonic cells 
1 Table 3 Differentially expressed genes in biofilm formation related KEGG pathways in L. plantarum RS66CD 2 biofilm compared to planktonic cells

\begin{tabular}{|c|c|c|c|c|c|}
\hline Term & Gene ID & Name & $\log _{2} \mathrm{FC}$ & Type & Function \\
\hline \multicolumn{6}{|c|}{ D-Alanine metabolism } \\
\hline & gene 1728 & $d l t A$ & 1.32 & up & D-alanine ligase \\
\hline & gene 2020 & $d d l$ & 1.39 & up & D-ala ligase \\
\hline & gene 433 & alr & 1.69 & up & Alanine racemase \\
\hline & gene1726 & $d l t C$ & 1.37 & up & D-alanine ligase \\
\hline \multicolumn{6}{|c|}{ Peptidoglycan biosynthesis } \\
\hline & gene422 & murA & 1.44 & up & Carboxyvinyl transferase \\
\hline & gene1094 & $d a c A$ & 2.49 & up & D-alanyl-D-alanine \\
\hline & & & & & Carboxypeptidase \\
\hline & gene1877 & $\operatorname{mraY}$ & 1.65 & up & Pentapeptide transferase \\
\hline & gene 2020 & $d d l$ & 1.39 & up & $\begin{array}{l}\text { D-ala ligase N-terminal domain } \\
\text { protein }\end{array}$ \\
\hline & gene707 & murB & 1.20 & up & UDP-pyruvate reductase \\
\hline & gene1075 & $l p-1256$ & 1.59 & up & Hypothetical protein \\
\hline \multicolumn{6}{|c|}{ Two-component system } \\
\hline & gene508 & lytR & 1.76 & up & LytR family transcriptional regulator \\
\hline & gene1358 & $g \ln A$ & -1.70 & down & Type I glutamate-ammonia ligase \\
\hline & gene939 & $c i t G$ & 1.39 & up & $\begin{array}{l}\text { Triphosphoribosyl-dephospho-CoA } \\
\text { synthase }\end{array}$ \\
\hline & gene 455 & $f t s H$ & 1.43 & up & ATP-dependent metallopeptidase \\
\hline & gene3028 & $\operatorname{lam} A$ & 1.51 & up & DNA-binding response regulator \\
\hline & gene2569 & $l p-3019$ & 2.15 & up & Membrane protein \\
\hline & gene 2757 & $\operatorname{lrg} B$ & 1.63 & up & Murein hydrolase \\
\hline & gene 2756 & $\operatorname{lrg} A$ & 2.65 & up & Murein hydrolase \\
\hline & gene3031 & $\operatorname{lam} B$ & 1.89 & up & Accessory gene regulator AgrB \\
\hline & gene 2603 & $h p k$ & 2.02 & up & Histidine protein kinase \\
\hline & gene635 & pstF & -1.28 & down & $\begin{array}{l}\text { Phosphate } \mathrm{ABC} \text { transporter } \\
\text { substrate-binding protein }\end{array}$ \\
\hline & gene963 & $\operatorname{cydA}$ & -1.93 & down & Cytochrome D ubiquinol oxidase \\
\hline & gene957 & mleS & 4.65 & up & NAD-dependent malic enzyme \\
\hline & gene 2658 & $l p-3125$ & 3.52 & up & Oxidoreductase \\
\hline & gene964 & $c y d B$ & -1.79 & down & Cytochrome d ubiquinol oxidase \\
\hline & gene682 & $\operatorname{rpoN}$ & -1.90 & down & RNA polymerase factor \\
\hline & gene4 & $\operatorname{gyrB}$ & 0.83 & up & Ica regulatory factor \\
\hline \multicolumn{6}{|c|}{ Carbon metabolism } \\
\hline & gene2989 & tkt4 & -1.99 & down & Transketolase \\
\hline & gene418 & $s d h B$ & 1.44 & up & L-serine dehydratase \\
\hline & gene419 & $\operatorname{sdh} A$ & 1.31 & up & L-serine dehydratase \\
\hline
\end{tabular}




\begin{tabular}{|c|c|c|c|c|c|}
\hline & gene163 & $\operatorname{ser} C$ & 1.93 & up & Phosphoserine aminotransferase \\
\hline & gene 162 & $\operatorname{ser} A$ & 1.80 & up & $\begin{array}{l}\text { D-3-phosphoglycerate } \\
\text { dehydrogenase }\end{array}$ \\
\hline & gene 1635 & pps & -1.58 & down & Phosphoenolpyruvate synthase \\
\hline & gene 1840 & $p d h C$ & -1.59 & down & $\begin{array}{l}\text { Branched-chain } \alpha \text {-keto acid } \\
\text { dehydrogenase }\end{array}$ \\
\hline & gene1841 & $p d h B$ & -1.50 & down & $\alpha$-keto acid dehydrogenase \\
\hline & gene 1842 & $p d h A$ & -1.19 & down & Pyruvate dehydrogenase \\
\hline & gene1839 & $p d h D$ & -1.51 & down & Dihydrolipoyl dehydrogenase \\
\hline & gene1534 & $\operatorname{pgm5}$ & -1.17 & down & Histidine phosphatase family protein \\
\hline \multicolumn{6}{|l|}{ Bacterial secretion system } \\
\hline & gene 3155 & $\operatorname{traK}$ & 2.47 & up & Conjugal transfer protein \\
\hline & gene 517 & $\operatorname{rad} A$ & 1.64 & up & DNA repair protein $\operatorname{RadA}$ \\
\hline & gene906 & $\sec Y$ & 1.35 & up & Preprotein translocase subunit \\
\hline
\end{tabular}




\section{Table 4 (on next page)}

Table 4 Differentially expressed biofilm formation related genes in L. plantarum RS66CD biofilm compared to planktonic cells 
1 Table 4 Differentially expressed biofilm formation related genes in L. plantarum RS66CD biofilm compared 2 to planktonic cells

\begin{tabular}{lllll}
\hline Gene ID & Name & $\log _{2} \mathrm{FC}$ & Type & Function \\
\hline gene747 & lp-0862 & -1.32 & down & Alcohol dehydrogenase \\
gene2442 & dapE & -1.71 & down & Metallo-hydrolase \\
gene1604 & dapB & -1.18 & down & 4-hydroxy-tetrahydrodipicolinate \\
& & & & reductase \\
gene1139 & pepV & -1.43 & down & Putative dipeptidase PepV \\
gene632 & groEL & 2.03 & up & chaperonin GroEL \\
gene1439 & fabG & 1.40 & up & SDR family oxidoreductase \\
gene1438 & fabD & 1.33 & up & Malonyltransferase \\
gene1435 & fabZ & 1.25 & up & dehydratase FabZ \\
gene1436 & fabH & 1.33 & up & 3-oxoacyl-ACP synthase III \\
\hline
\end{tabular}

$3 \quad * \log _{2}$ FC: fold change in L. plantarum RS66CD biofilm compared to planktonic cells 


\section{Figure 1}

Figure 1 Quantification of L. plantarum RS66CD biofilm at different $\mathrm{NaCl}$ concentrations

The data columns represent means of the three repetitions. The error bars show standard deviation. Different lowercase letters indicate significant difference $(p<0.05)$.

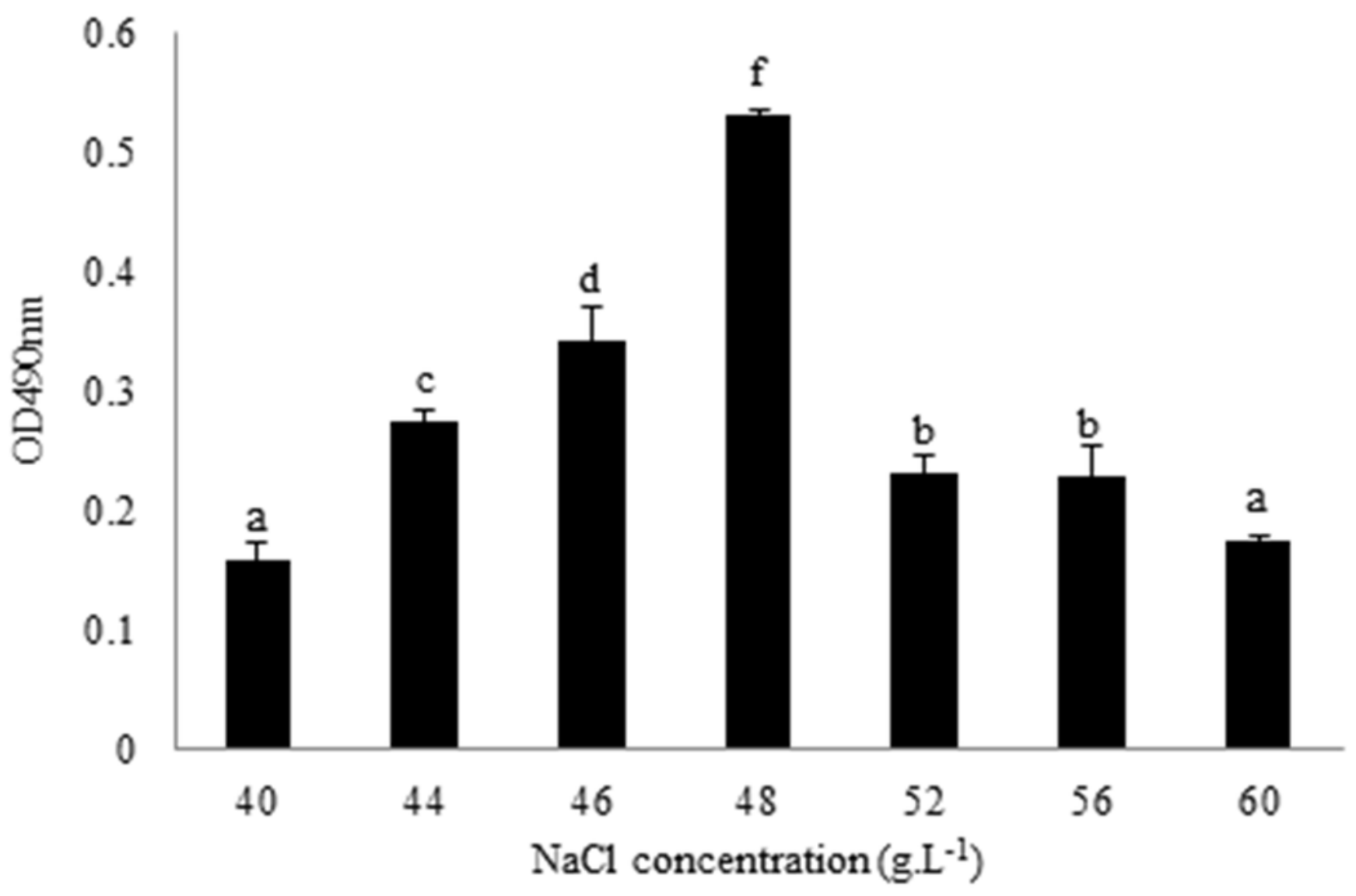




\section{Figure 2}

Figure 2 SEM micrographs of $L$. plantarum RS66CD

SEM micrographs of $L$. plantarum RS66CD grown in a) modified MRS medium with $\mathrm{NaCl}$ and

b) MRS medium
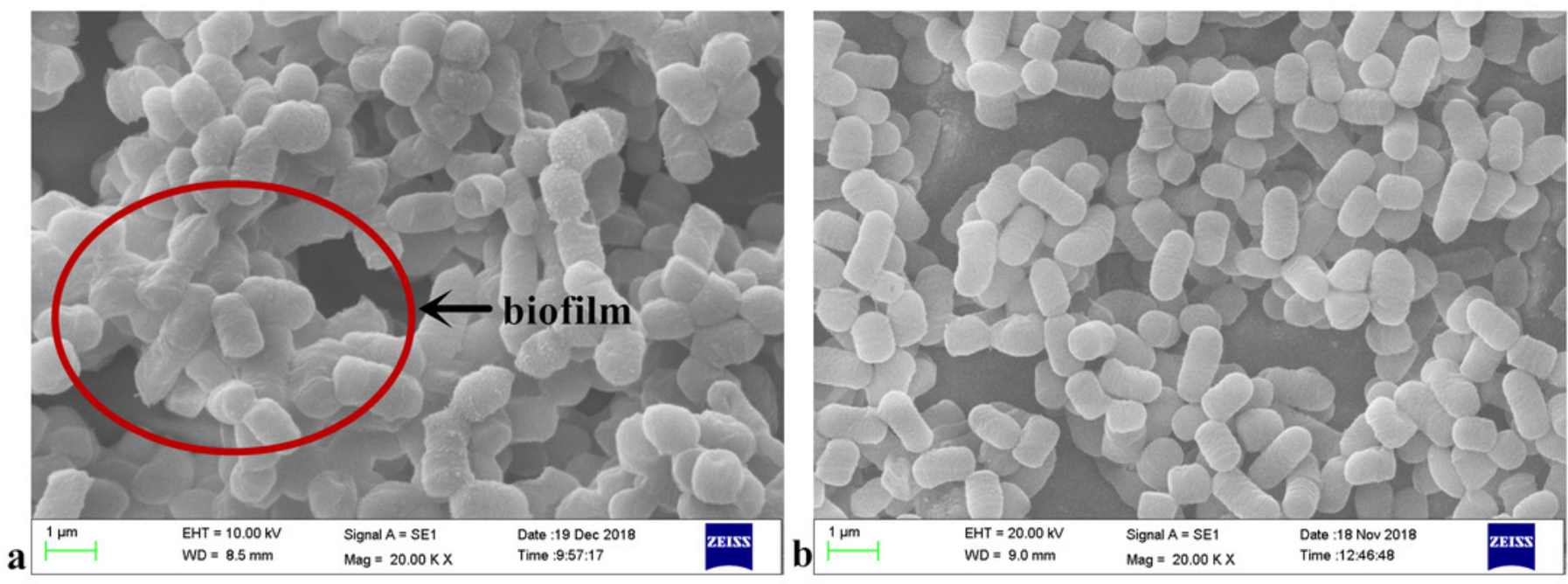
Figure 3

Figure 3 Differentially expressed genes in L. plantarum RS66CD biofilm compared to planktonic cells

* Log $_{2} \mathrm{FC}$ : fold change in L. plantarum RS66CD biofilm compared to planktonic cells

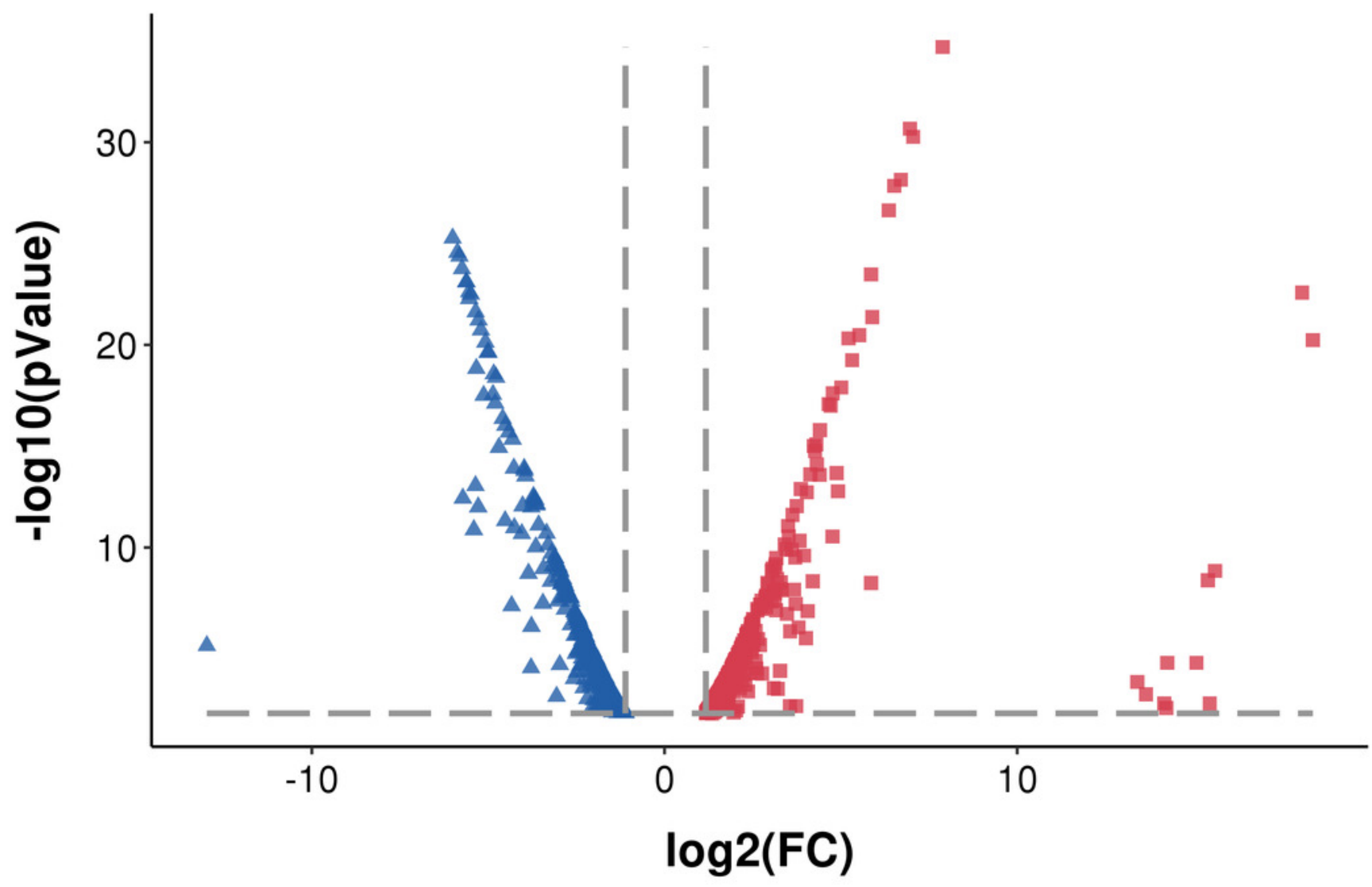

Significance - up: 447 no_diff: 0 × down: 426 


\section{Figure 4}

Figure 4 KEGG pathway analysis of differentially expressed genes in L. plantarum RS66CD biofilm compared to planktonic cells.

The enriched KEGG categories are on the vertical axis. The enrichment factor, i.e. the ratio of the enriched DEGs in the KEGG category to the total genes in that category, is shown on the horizontal axis

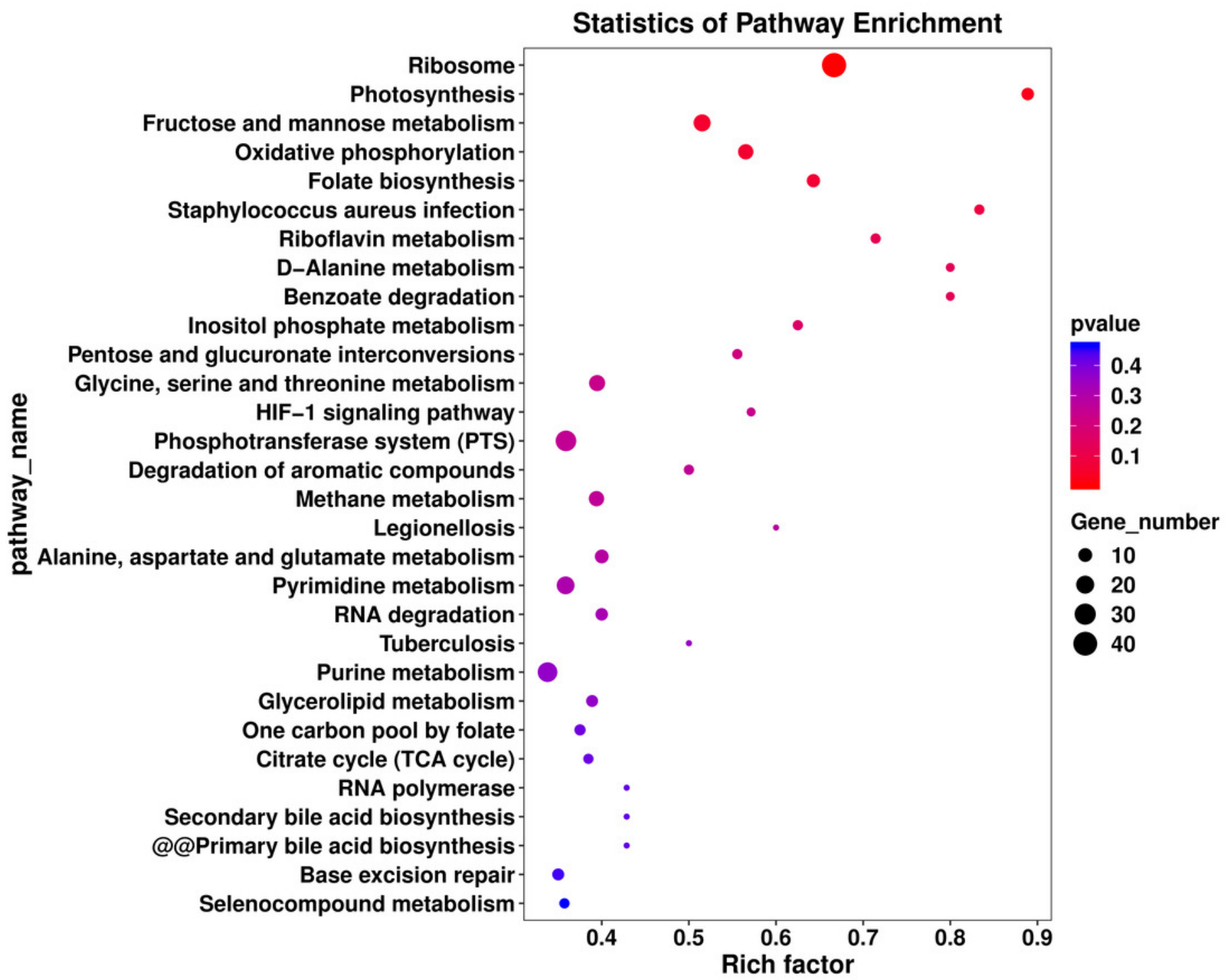


Figure 5

Figure 5 Expression of differentially expressed biofilm related genes in L. plantarum RS66CD

(Samples in columns and genes on rows. P, planktonic cells; B, biofilm. Differential expression is indicated with the color-coding explained in the top right corner) 


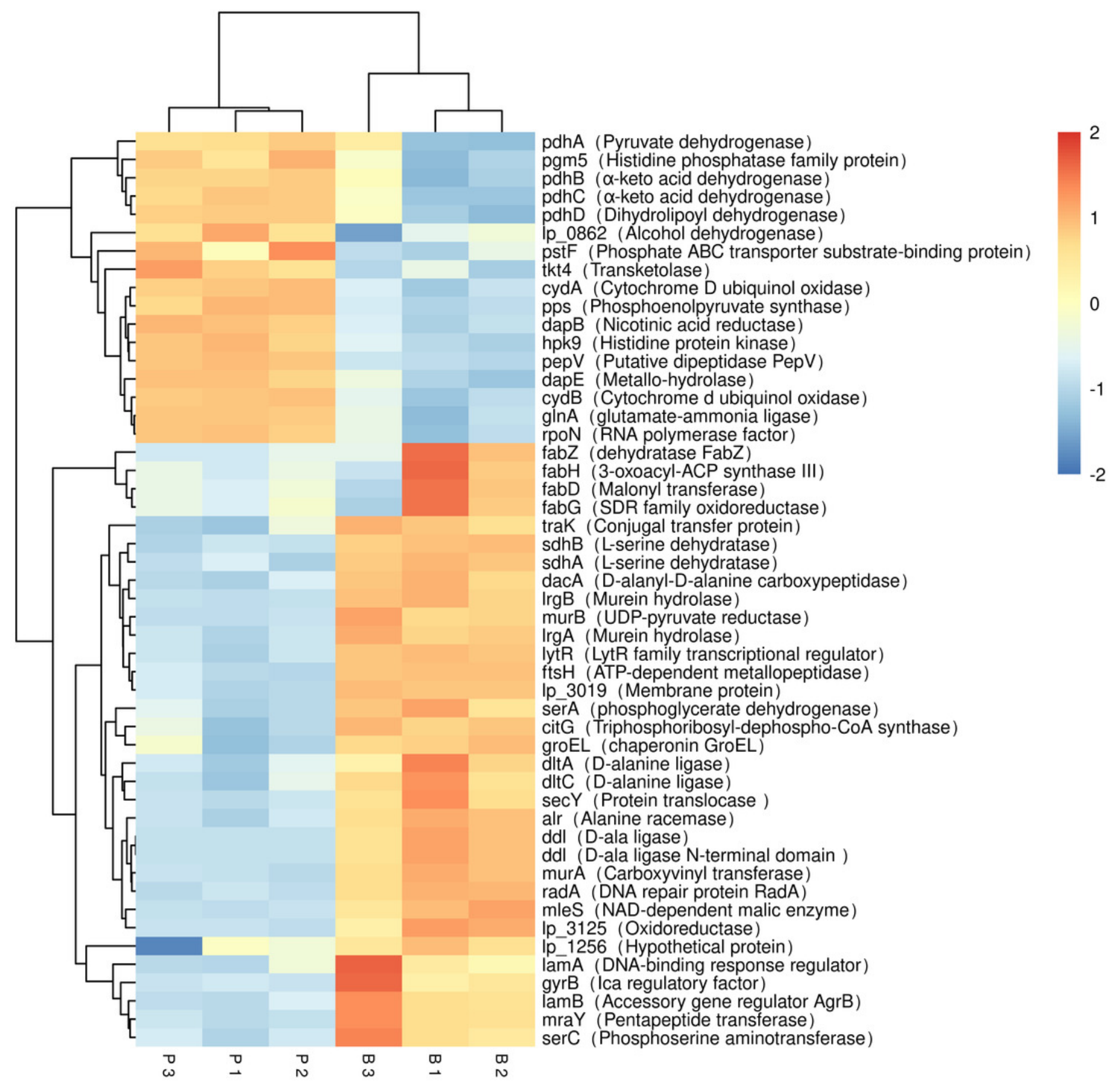

